DRAFT VERSION OCTOBER 9, 2018

Typeset using LATEX manuscript style in AASTeX62

\title{
PARALLEL AND PERPENDICULAR DIFFUSION COEFFICIENTS OF ENERGETIC CHARGED PARTICLES WITH ADIABATIC FOCUSING
}

\author{
J. F. WANG ${ }^{1}$ AND G. QIN ${ }^{1}$ \\ ${ }^{1}$ School of Science, Harbin Institute of Technology, Shenzhen, 518055, China; qingang@ @it.edu.cn
}

\begin{abstract}
It is very important to understand stochastic diffusion of energetic charged particles in nonuniform background magnetic field in plasmas of astrophysics and fusion devices. Using different methods considering along-field adiabatic focusing effect, various authors derived parallel diffusion coefficient $\kappa_{\|}$and its correction $T$ to $\kappa_{\| 0}$, where $\kappa_{\| 0}$ is the parallel diffusion coefficient without adiabatic focusing effect. In this paper, using the improved perturbation method developed by He \& Schlickeiser and iteration process, we obtain a new correction $T^{\prime}$ to $\kappa_{\| 0}$. Furthermore, by employing the isotropic pitch-angle scattering model $D_{\mu \mu}=D\left(1-\mu^{2}\right)$, we find that $T^{\prime}$ has the different sign as that of $T$. In this paper the spatial perpendicular diffusion coefficient $\kappa_{\perp}$ with the adiabatic focusing effect is also obtained.
\end{abstract}

Keywords: diffusion, magnetic fields, scattering, turbulence

\section{INTRODUCTION}

Energetic charged particle propagation in magnetic turbulent field is one of the fundamental problems in astrophysics (e.g., cosmic ray physics, astrophysical plasmas, and space weather research) and Tokamak fusion devices (see, e.g., Jokipii 1966; Schlickeiser 2002; Matthaeus et al. 2003; Shalchi \& Schlickeiser

Corresponding author: G. Qin

qingang@hit.edu.cn 
2005; Shalchi et al. 2006; Qin 2007; Hauff \& Jenko 2008; Shalchi 2009a, 2010; Qin \& Zhang 2014). The magnetic turbulence can cause the field line wandering, or the field line random walk (FLRW) (Jokipii 1966; Matthaeus et al. 1995; Shalchi \& Kourakis 2007; Shalchi \& Qin 2010; Wang et al. 2017a), which directly affects the diffusion of charged particles. In the investigation of energetic particle transport through magnetized plasma, according to observations one usually assumes the magnetic field configuration as the superposition of a background magnetic field $B_{0}$ and a turbulent component $\delta \vec{B}$. Because the background magnetic field breaks the symmetry of the magnetized plasma, one have to distinguish particle diffusion along and across the large-scale magnetic field. However, some previous articles only consider the parallel diffusion, since it is much greater than the perpendicular one in many scenarios (see, e.g., Earl 1974, 1976; Beeck \& Wibberenz 1986; Shalchi 2011; Litvinenko 2012a,b; Shalchi \& Danos 2013; He \& Schlickeiser 2014). On the other hand, in some conditions, e.g., with high turbulence levels, perpendicular diffusion of energetic particles becomes too important to be ignored (Dwyer et al. 1997; Zhang et al. 2003).

Various analytical theories of parallel and perpendicular diffusion for energetic charged particles have been developed in the past. Jokipii (1966) established the quasi-linear theory of diffusion which corresponds to the first order perturbation theory. However, the quasi-linear theory is problematic and nonlinear description for particle propagation is essential, especially for particle perpendicular diffusion (e.g., Qin et al. 2002). NonLinear Guiding Center (NLGC) theory developed by Matthaeus et al. (2003) is the important breakthrough, which agrees well with the computer simulations of two-component (slab+two-dimensional) turbulence model (see, Matthaeus et al. 1990). Based on NLGC, Shalchi (2010) developed the Unified NonLinear Transport (UNLT) theory for perpendicular diffusion coefficient, using the Fokker-Planck equation of energetic charged particles to treat the fourth-order correlation. It is suggested that UNLT can be applied for arbitrary turbulence geometry to explain the subdiffusive transport for slab turbulence and the recovery of diffusion for three-dimensional and quasi-three-dimensional turbulence models.

By radio continuum surveys of interstellar space and direct in-situ measurements in solar system, it is well established that for many scenarios the background magnetic fields are spatially varying. However, the above research about parallel and perpendicular diffusion only explored the uniform mean magnetic field. One can show that the spatially varying background magnetic fields lead to the adiabatic focusing effect 
of charged energetic particle transport and introduces correction to the particle diffusion coefficients (see, e.g., Roelof 1969; Earl 1976; Kunstmann 1979; Beeck \& Wibberenz 1986; Bieber \& Burger 1990; Kóta 2000; Schlickeiser \& Shalchi 2008; Shalchi 2009b, 2011; Litvinenko 2012a,b; Shalchi \& Danos 2013; Wang \& Qin 2016; Wang et al. 2017b). To explore the influence of adiabatic focusing on particle transport, perturbation method is frequently used (see, e.g., Beeck \& Wibberenz 1986; Bieber \& Burger 1990; Schlickeiser \& Shalchi 2008; Schlickeiser \& Jenko 2010; Litvinenko \& Schlickeiser 2013; He \& Schlickeiser 2014). To use the perturbation method, since anisotropic distribution function is an implicit function, by using iteration method one can find that the anisotropic distribution function becomes infinite series of the spatial and temporal derivatives of the isotropic distribution function. Therefore, the governing equation of isotropic distribution function derived from the Fokker-Planck equation contains infinite terms because of the infinite series of anisotropic distribution function. By using truncating method to neglect the higherorder derivative terms, the approximate correction formulas of parallel or perpendicular diffusion coefficients were obtained (see, e.g., Schlickeiser \& Shalchi 2008; Schlickeiser \& Jenko 2010; He \& Schlickeiser 2014). However, the higher-order derivative terms probably also make the correction to the parallel and perpendicular diffusion as the lower-order derivative ones. Maybe the magnitude of the correction from the higher-order derivative terms might not be the higher order small quantity than that of the correction from the lower-order derivative terms. Therefore, the correction obtained by the previous authors is likely to contain significant error. In this paper by considering the higher-order derivative terms we derive the parallel and perpendicular diffusion coefficients and obtain the correction formulas coming from all order derivative terms by using the improved perturbation method (He \& Schlickeiser 2014) and the iteration operation. And for the weak adiabatic focusing limit we evaluate the correction to the parallel diffusion coefficient and compare it with the correction obtained in the previous papers.

The paper is organized as follows. In Section 2, by considering adiabatic focusing effect, we derive the governing equation of isotropic distribution function with the infinite series. In Section 3, by employing the truncation, we deduce the approximate formulas of the perpendicular and parallel diffusion coefficients, and that of the streaming term. In Section 4, the parallel and perpendicular diffusion coefficients $\kappa_{\|}$and $\kappa_{\perp}$, 
and the parallel streaming coefficient $\kappa_{1}$ are derived, with the influence of the infinite series caused by the iteration of anisotropic distribution function. We conclude and summarize our results in Section 5.

\section{EQUATION OF ISOTROPIC DISTRIBUTION FUNCTION}

The starting point of this paper is the modified Fokker-Planck equation for the gyro-tropic energetic charged particle distribution function, which incorporates the pitch-angle and perpendicular diffusion, and the along-field adiabatic focusing

$$
\frac{\partial f}{\partial t}+v \mu \frac{\partial f}{\partial z}=\frac{\partial}{\partial \mu}\left[D_{\mu \mu} \frac{\partial f}{\partial \mu}-\frac{v}{2 L}\left(1-\mu^{2}\right) f\right]+D_{\perp} \Delta_{\perp} f .
$$

Here $t$ is time, $z$ is the distance along the background magnetic field, $\mu=v_{z} / v$ is the pitch-angle cosine with particle speed $v$ and its z-component $v_{z}, D_{\mu \mu}$ is the pitch-angle diffusion coefficient, $D_{\perp}$ is the Fokker-Planck perpendicular diffusion coefficient, $L(z)=-B_{0}(z) /\left[d B_{0}(z) / d z\right]$ is the adiabatic focusing characteristic length of the large-scale magnetic field $B_{0}(z)$, and $\Delta_{\perp}=\partial^{2} / \partial x^{2}+\partial^{2} / \partial y^{2}$ is the differential operator across the largescale magnetic field. The source term is not included in the above equation. Because only the influence of the along-field adiabatic focusing on the parallel and perpendicular diffusion is explored in this paper, the terms related to momentum diffusion and so on are ignored in the Fokker-Planck Equation (1). The more complete form of the Fokker-Planck equation can be found in Schlickeiser (2002).

It should be mentioned that the linear phase space density $f(x, y, z, p, \mu, t)=f_{0}(x, y, z, p, \mu, t) / B_{0}(z)$, so that Equation (1) is equivalent to the standard Fokker-Planck equation

$$
\frac{\partial f_{0}}{\partial t}+v \mu \frac{\partial f_{0}}{\partial z}=\frac{\partial}{\partial \mu}\left[D_{\mu \mu} \frac{\partial f_{0}}{\partial \mu}\right]-\frac{v}{2 L}\left(1-\mu^{2}\right) \frac{\partial f_{0}}{\partial \mu}+D_{\perp} \Delta_{\perp} f_{0}
$$

If the pitch-angle scattering is strong, the gyro-tropic phase space distribution function quickly becomes quasi-equilibrium distribution. Therefore, we can split the gyro-tropic cosmic-ray phase space density $f(\vec{x}, \mu, t)$ into the dominant isotropic part $F(\vec{x}, t)$ and the subordinate anisotropic part $g(\vec{x}, \mu, t)$ as following the previous articles (see, e.g., Schlickeiser et al 2007; Schlickeiser \& Shalchi 2008; He \& Schlickeiser 2014)

$$
f(\vec{x}, \mu, t)=F(\vec{x}, t)+g(\vec{x}, \mu, t)
$$


with

$$
F(\vec{x}, t)=\frac{1}{2} \int_{-1}^{1} d \mu f(\vec{x}, \mu, t)
$$

and

$$
\int_{-1}^{1} d \mu g(\vec{x}, \mu, t)=0
$$

\subsection{The differential equation of anisotropic distribution function $g(\mu)$}

In this subsection, we would adopt the method in He \& Schlickeiser (2014) to derive the differential equation of the anisotropic distribution function $g(\mu)$.

By integrating Equation (1) over $\mu$ from -1 to 1 we can obtain

$$
\frac{\partial F}{\partial t}+\frac{v}{2} \frac{\partial}{\partial z} \int_{-1}^{1} \mu g d \mu=\frac{1}{2} \Delta_{\perp} F \int_{-1}^{1} d \mu D_{\perp}+\frac{1}{2} \Delta_{\perp} \int_{-1}^{1} d \mu D_{\perp} g .
$$

Furthermore, by integrating Equation (1) over $\mu$ from -1 to $\mu$, the following equation can be found

$$
\begin{aligned}
\frac{\partial F}{\partial t}(\mu+1) & +\frac{\partial}{\partial t} \int_{-1}^{\mu} d \mu g+\frac{v\left(\mu^{2}-1\right)}{2} \frac{\partial F}{\partial z}+v \frac{\partial}{\partial z} \int_{-1}^{\mu} d \mu \mu g \\
& =D_{\mu \mu} \frac{\partial g}{\partial \mu}-\frac{v\left(1-\mu^{2}\right)}{2 L} F-\frac{v\left(1-\mu^{2}\right)}{2 L} g+\Delta_{\perp} \int_{-1}^{\mu} d \mu D_{\perp} F+\Delta_{\perp} \int_{-1}^{\mu} d \mu D_{\perp} g .
\end{aligned}
$$

To obtain the Equations (6) and (7) the assumption $D_{\mu \mu}(\mu= \pm 1)=0$ is used. Subtracting Equation (6) from (7) we can get

$$
\frac{\partial g}{\partial \mu}-\frac{v\left(1-\mu^{2}\right) g}{2 L D_{\mu \mu}}+\frac{v\left(1-\mu^{2}\right)}{2 D_{\mu \mu}}\left(\frac{\partial F}{\partial z}-\frac{F}{L}\right)=\Phi(\mu)
$$

with

$$
\begin{aligned}
\Phi(\mu)= & \frac{1}{D_{\mu \mu}}\left[\left(\frac{\partial F}{\partial t} \mu+\frac{\partial}{\partial t} \int_{-1}^{\mu} g d v\right)-\Delta_{\perp}\left(\int_{-1}^{\mu} d v D_{\perp} g-\frac{1}{2} \int_{-1}^{1} d \mu D_{\perp} g\right)\right. \\
& \left.+\frac{v}{2} \frac{\partial}{\partial z}\left(2 \int_{-1}^{\mu} d v v g-\int_{-1}^{1} d \mu \mu g\right)-\Delta_{\perp} F\left(\int_{-1}^{\mu} d v D_{\perp}-\frac{1}{2} \int_{-1}^{1} d \mu D_{\perp}\right)\right] .
\end{aligned}
$$

By defining the following quantity

$$
M(\mu)=\frac{v}{2 L} \int_{-1}^{\mu} d v \frac{1-v^{2}}{D_{\mu \mu}(v)}
$$

we can obtain

$$
\frac{\partial}{\partial \mu}\left\{\left[g(\mu)-L\left(\frac{\partial F}{\partial z}-\frac{F}{L}\right)\right] e^{-M(\mu)}\right\}=e^{-M(\mu)} \Phi(\mu) .
$$

It is noted that in He \& Schlickeiser (2014) the right hand side of this equation (Equation (20) in their paper) is set to be 0 . 


\subsection{The integration results of $g(\mu)$}

Through integrating Equation (11) over $\mu$, we can get the anisotropic distribution function as the following

$$
g(\mu)=L\left(\frac{\partial F}{\partial z}-\frac{F}{L}\right)\left[1-\frac{2 e^{M(\mu)}}{\int_{-1}^{1} d \mu e^{M(\mu)}}\right]+e^{M(\mu)}\left[R(\mu)-\frac{\int_{-1}^{1} d \mu e^{M(\mu)} R(\mu)}{\int_{-1}^{1} d \mu e^{M(\mu)}}\right]
$$

with

$$
R(\mu)=\int_{-1}^{\mu} d v e^{-M(v)} \Phi(v) .
$$

Equation (12) contains the effect from the term on the right-hand side of Equation (11), whereas the Equation (20) in He \& Schlickeiser (2014) is an approximate expression.

Inserting $R(\mu)$ (Equation (13)) into $g(\mu)$ (Equation (12)) and considering Equation (9), we find that the anisotropic distribution function $g(\mu)$ becomes

$$
\begin{aligned}
g(\mu)= & L\left(\frac{\partial F}{\partial z}-\frac{F}{L}\right)\left[1-\frac{2 e^{M(\mu)}}{\int_{-1}^{1} d \mu e^{M(\mu)}}\right]+e^{M(\mu)}\left\{\int _ { - 1 } ^ { \mu } d v e ^ { - M ( v ) } \frac { 1 } { D _ { \mu \mu } } \left[\left(\frac{\partial F}{\partial t} \mu+\frac{\partial}{\partial t} \int_{-1}^{\mu} g d v\right)\right.\right. \\
& \left.-\Delta_{\perp}\left(\int_{-1}^{\mu} d v D_{\perp} g-\frac{1}{2} \int_{-1}^{1} d \mu D_{\perp} g\right)+\frac{v}{2} \frac{\partial}{\partial z}\left(2 \int_{-1}^{\mu} d v v g-\int_{-1}^{1} d \mu \mu g\right)-\Delta_{\perp} F\left(\int_{-1}^{\mu} d v D_{\perp}-\frac{1}{2} \int_{-1}^{1} d \mu D_{\perp}\right)\right] \\
& -\frac{1}{\int_{-1}^{1} d \mu e^{M(\mu)}} \int_{-1}^{1} d \mu e^{M(\mu)} \int_{-1}^{\mu} d v e^{-M(v)} \frac{1}{D_{\mu \mu}}\left[\left(\frac{\partial F}{\partial t} \mu+\frac{\partial}{\partial t} \int_{-1}^{\mu} g d v\right)-\Delta_{\perp}\left(\int_{-1}^{\mu} d v D_{\perp} g-\frac{1}{2} \int_{-1}^{1} d \mu D_{\perp} g\right)\right. \\
& \left.\left.+\frac{v}{2} \frac{\partial}{\partial z}\left(2 \int_{-1}^{\mu} d v v g-\int_{-1}^{1} d \mu \mu g\right)-\Delta_{\perp} F\left(\int_{-1}^{\mu} d v D_{\perp}-\frac{1}{2} \int_{-1}^{1} d \mu D_{\perp}\right)\right]\right\} .
\end{aligned}
$$

From the latter equation we can find that the anisotropic distribution function $g(\mu)$ is an implicit function. Therefore, by iterating Equation (14), i.e. applying it repeatedly, we obtain the formula of $g(\mu)$ as

$$
g(\mu)=\sum_{m, n, p} \epsilon_{m, n, p} \frac{\partial^{m+n}}{\partial t^{m} \partial z^{n}} \Delta_{\perp}^{p} F
$$

with the coefficients $\epsilon_{m, n, p}$. Here $m, n, p=0,1,2,3, \cdots$. Similarily, by inserting Equation (12) into Equation (9) and inputting Equation (9) into Equation (13), we can find that $R(\mu)$ is also an implicit function. Therefore, with iteration operation $R(\mu)$ can also be written as

$$
R(\mu)=\sum_{m, n, p} \chi_{m, n, p} \frac{\partial^{m+n}}{\partial t^{m} \partial z^{n}} \Delta_{\perp}^{p} F
$$

with the coefficients $\chi_{m, n, p}$ and $m, n, p=0,1,2,3, \cdots$. Apparently, the coefficients $\chi_{m, n, p}$ in the latter equation is related to the coefficients $\epsilon_{m, n, p}$ in Equation (15) since $g(\mu)$ and $R(\mu)$ are related according to Equations (9), (12), (13), and (14). 


\subsection{The governing equation of the isotropic distribution function $F(\vec{x}, t)$}

From Equation (6) we can find that in order to obtain the governing equation of isotropic distribution function $F(\vec{x}, t)$ we have to get the expression of $\int_{-1}^{1} d \mu \mu g$ and $\int_{-1}^{1} d \mu D_{\perp} g$. By using Equation (12) we can obtain

$$
\int_{-1}^{1} d \mu \mu g=-2 \frac{\int_{-1}^{1} d \mu \mu e^{M(\mu)}}{\int_{-1}^{1} d \mu e^{M(\mu)}}\left(\frac{\partial F}{\partial z}-\frac{F}{L}\right) L+\int_{-1}^{1} d \mu \mu e^{M(\mu)}\left[R(\mu)-\frac{\int_{-1}^{1} d \mu e^{M(\mu)} R(\mu)}{\int_{-1}^{1} d \mu e^{M(\mu)}}\right]
$$

and

$$
\int_{-1}^{1} d \mu D_{\perp} g=L\left(\frac{\partial F}{\partial z}-\frac{F}{L}\right) \int_{-1}^{1} d \mu D_{\perp}\left[1-\frac{2 e^{M(\mu)}}{\int_{-1}^{1} d \mu e^{M(\mu)}}\right]+\int_{-1}^{1} d \mu D_{\perp} e^{M(\mu)}\left[R(\mu)-\frac{\int_{-1}^{1} d \mu e^{M(\mu)} R(\mu)}{\int_{-1}^{1} d \mu e^{M(\mu)}}\right]
$$

Substituting Equations (17) and (18) into Equation (6) yields

$$
\begin{gathered}
\frac{\partial F}{\partial t}-\frac{\partial}{\partial z}\left[v L \frac{\int_{-1}^{1} d \mu \mu e^{M(\mu)}}{\int_{-1}^{1} d \mu e^{M(\mu)}}\left(\frac{\partial F}{\partial z}-\frac{F}{L}\right)\right]-\Delta_{\perp} F \frac{\int_{-1}^{1} d \mu D_{\perp} e^{M(\mu)}}{\int_{-1}^{1} d \mu e^{M(\mu)}} \\
-\frac{L}{2} \Delta_{\perp} \frac{\partial F}{\partial z} \int_{-1}^{1} d \mu D_{\perp}\left[1-\frac{2 e^{M(\mu)}}{\int_{-1}^{1} d \mu e^{M(\mu)}}\right]=\Lambda(x, y, z, t)
\end{gathered}
$$

with

$$
\Lambda(x, y, z, t)=-\frac{v}{2} \int_{-1}^{1} d \mu \mu e^{M(\mu)}\left[\frac{\partial R}{\partial z}-\frac{\int_{-1}^{1} d \mu \frac{\partial R}{\partial z} e^{M(\mu)}}{\int_{-1}^{1} d \mu e^{M(\mu)}}\right]+\frac{1}{2} \int_{-1}^{1} d \mu D_{\perp} e^{M(\mu)}\left[\Delta_{\perp} R-\frac{\int_{-1}^{1} d \mu e^{M(\mu)} \Delta_{\perp} R}{\int_{-1}^{1} d \mu e^{M(\mu)}}\right] .
$$

By inserting Equation (16) into the latter equation we can obtain the following formula

$$
\Lambda(x, y, z, t)=\sum_{m, n, p} \eta_{m, n, p} \frac{\partial^{m+n}}{\partial t^{m} \partial z^{n}} \Delta_{\perp}^{p} F
$$

with the coefficients $\eta_{m, n, p}$ and $m, n, p=0,1,2,3, \cdots$. Note that some of the coefficients $\eta_{m, n, p}$ are equal to 0 .

Replacing $\Lambda(x, y, z, t)$ in Equation (19) with the latter equation gives

$$
\begin{aligned}
\frac{\partial F}{\partial t}-\frac{\partial}{\partial z} & {\left[v L \frac{\int_{-1}^{1} d \mu \mu e^{M(\mu)}}{\int_{-1}^{1} d \mu e^{M(\mu)}}\left(\frac{\partial F}{\partial z}-\frac{F}{L}\right)\right]-\Delta_{\perp} F \frac{\int_{-1}^{1} d \mu D_{\perp} e^{M(\mu)}}{\int_{-1}^{1} d \mu e^{M(\mu)}} } \\
& -\frac{L}{2} \Delta_{\perp} \frac{\partial F}{\partial z} \int_{-1}^{1} d \mu D_{\perp}\left[1-\frac{2 e^{M(\mu)}}{\int_{-1}^{1} d \mu e^{M(\mu)}}\right]=\sum_{m, n, p} \eta_{m, n, p} \frac{\partial^{m+n}}{\partial t^{m} \partial z^{n}} \Delta_{\perp}^{p} F
\end{aligned}
$$

The latter equation is the most general resulting transport equation in this paper. 
After combining similar terms for the latter equation, we can obtain

$$
\begin{aligned}
\left(\eta_{1,0,0}+1\right) \frac{\partial F}{\partial t} & +\left(\eta_{0,1,0}+v \frac{\int_{-1}^{1} d \mu \mu e^{M(\mu)}}{\int_{-1}^{1} d \mu e^{M(\mu)}}\right) \frac{\partial F}{\partial z}=\left(\eta_{0,2,0}+v L \frac{\int_{-1}^{1} d \mu \mu e^{M(\mu)}}{\int_{-1}^{1} d \mu e^{M(\mu)}}\right) \frac{\partial^{2} F}{\partial z^{2}}+\left(\eta_{0,0,1}+\frac{\int_{-1}^{1} d \mu D_{\perp} e^{M(\mu)}}{\int_{-1}^{1} d \mu e^{M(\mu)}}\right) \Delta_{\perp} F \\
& +\left(\eta_{0,1,1}+\frac{L}{2} \int_{-1}^{1} d \mu D_{\perp}\left[1-\frac{2 e^{M(\mu)}}{\int_{-1}^{1} d \mu e^{M(\mu)}}\right]\right) \Delta_{\perp} \frac{\partial F}{\partial z}+\sum_{(m, n, p) \notin A} \eta_{m, n, p} \frac{\partial^{m+n}}{\partial t^{m} \partial z^{n}} \Delta_{\perp}^{p} F
\end{aligned}
$$

Here, $A=\{(1,0,0),(0,1,0),(0,2,0),(0,0,1),(0,1,1)\}$.

Furthermore, Equation (23) can be simply rewritten as

$$
\kappa_{1,0,0} \frac{\partial F}{\partial t}+\kappa_{0,1,0} \frac{\partial F}{\partial z}=\sum_{(m, n, p) \notin B} \kappa_{m, n, p} \frac{\partial^{m+n}}{\partial t^{m} \partial z^{n}} \Delta_{\perp}^{p} F
$$

with

$$
\kappa_{m, n, p}= \begin{cases}\eta_{1,0,0}+1, & (m, n, p)=(1,0,0) \\ \eta_{0,1,0}+v \frac{\int_{-1}^{1} d \mu \mu e^{M(\mu)}}{\int_{-1}^{1} d \mu e^{M(\mu)}}, & (m, n, p)=(0,1,0) \\ \eta_{0,0,1}+\frac{\int_{-1}^{1} d \mu D_{\perp} e^{M(\mu)}}{\int_{-1}^{1} d \mu e^{M(\mu)}}, & (m, n, p)=(0,0,1) \\ \eta_{0,2,0}+v L \frac{\int_{-1}^{1} d \mu \mu e^{M(\mu)}}{\int_{-1}^{1} d \mu e^{M(\mu)}}, & (m, n, p)=(0,2,0) \\ \eta_{0,1,1}+\frac{L}{2} \int_{-1}^{1} d \mu D_{\perp}\left[1-\frac{2 e^{M(\mu)}}{\int_{-1}^{1} d \mu e^{M(\mu)}}\right], & (m, n, p)=(0,1,1) \\ \eta_{m, n, p} & \text { otherwise. }\end{cases}
$$

Here, $B=\{(1,0,0),(0,1,0)\}, \kappa_{1,0,0}$ is the coefficient of the first order time derivative term, $\kappa_{0,1,0}$ is the coefficient of parallel streaming term, $\kappa_{0,2,0}$ is the parallel diffusion coefficient, $\kappa_{0,0,1}$ is the perpendicular diffusion coefficient, and $\kappa_{0,1,1}$ is the coefficient of the term with $\Delta_{\perp} \partial F / \partial z$. Equation (23) and (24) are equivalent to Equation (22). They are all the forms of the governing equation of the isotropic distribution function in the most general case.

In this paper, we only explore the properties of the first order time derivative term coefficient $\kappa_{1,0,0}$, the parallel streaming coefficient $\kappa_{0,1,0}$, the parallel diffusion coefficient $\kappa_{0,2,0}$, and the perpendicular diffusion coefficient $\kappa_{0,0,1}$, which are listed as

$$
\begin{aligned}
& \kappa_{1,0,0}=\eta_{1,0,0}+\kappa_{1,0,0}^{a}, \\
& \kappa_{0,1,0}=\eta_{0,1,0}+\kappa_{0,1,0}^{a},
\end{aligned}
$$




$$
\begin{aligned}
& \kappa_{0,0,1}=\eta_{0,0,1}+\kappa_{0,0,1}^{a} \\
& \kappa_{0,2,0}=\eta_{0,2,0}+\kappa_{0,2,0}^{a},
\end{aligned}
$$

with

$$
\begin{aligned}
& \kappa_{1,0,0}^{a}=1 \\
& \kappa_{0,1,0}^{a}=v \frac{\int_{-1}^{1} d \mu \mu e^{M(\mu)}}{\int_{-1}^{1} d \mu e^{M(\mu)}} \\
& \kappa_{0,0,1}^{a}=\frac{\int_{-1}^{1} d \mu D_{\perp} e^{M(\mu)}}{\int_{-1}^{1} d \mu e^{M(\mu)}} \\
& \kappa_{0,2,0}^{a}=v L \frac{\int_{-1}^{1} d \mu \mu e^{M(\mu)}}{\int_{-1}^{1} d \mu e^{M(\mu)}} .
\end{aligned}
$$

Note that any of the coefficients $\eta_{1,0,0}, \eta_{0,1,0}, \eta_{0,0,1}$, and $\eta_{0,2,0}$ might be zero.

\section{ANALYTICAL COEFFICIENTS WITH $\Lambda(X, Y, Z, T)=0$}

For the condition $\Lambda(\vec{x}, \mu, t)=0$, from Equation (20) we can find that $R(\mu)=0$. So, from formula (12) the approximate anisotropic distribution function can be obtained

$$
g^{a}(\mu)=L\left(\frac{\partial F}{\partial z}-\frac{F}{L}\right)\left[1-\frac{2 e^{M(\mu)}}{\int_{-1}^{1} d \mu e^{M(\mu)}}\right],
$$

which is identical with the Equation (20) in He \& Schlickeiser (2014). In addition, by using the condition $\Lambda(\mu)=0$, i.e., setting $\eta_{m, n, p}=0$ for any $m, n, p$, we can simplify Equation (23) as

$$
\frac{\partial F}{\partial t}+\kappa_{1}^{a} \frac{\partial F}{\partial z}=\frac{\partial}{\partial z}\left(\kappa_{\|}^{a} \frac{\partial F}{\partial z}\right)+\kappa_{\perp}^{a} \Delta_{\perp} F+\kappa_{3}^{a} \Delta_{\perp} \frac{\partial F}{\partial z}
$$

with

$$
\begin{aligned}
& \kappa_{t}^{a}=\kappa_{1,0,0}^{a}=1 \\
& \kappa_{1}^{a}=\kappa_{0,1,0}^{a}=\frac{\kappa_{\|}}{L}=v \frac{\int_{-1}^{1} d \mu \mu e^{M(\mu)}}{\int_{-1}^{1} d \mu e^{M(\mu)}} \\
& \kappa_{\perp}^{a}=\kappa_{0,0,1}^{a}=\frac{\int_{-1}^{1} d \mu D_{\perp} e^{M(\mu)}}{\int_{-1}^{1} d \mu e^{M(\mu)}}
\end{aligned}
$$




$$
\begin{aligned}
& \kappa_{\|}^{a}=\kappa_{0,2,0}^{a}=v L \frac{\int_{-1}^{1} d \mu \mu e^{M(\mu)}}{\int_{-1}^{1} d \mu e^{M(\mu)}} \\
& \kappa_{3}^{a}=\frac{L}{2} \int_{-1}^{1} d \mu D_{\perp}\left[1-\frac{2 e^{M(\mu)}}{\int_{-1}^{1} d \mu e^{M(\mu)}}\right] .
\end{aligned}
$$

Obviously, Equations (36)-(40) are the special forms of Equations (26)-(28) with $\Lambda(x, y, z, t)=0$. In particular, Equation (38) shows the approximate perpendicular diffusion coefficient with the adiabatic focusing effect. Furthermore, the approximate parallel diffusion coefficient $\kappa_{\|}^{a}$ (Equation (39)) is identical with the result obtained by some previous authors (see, Beeck \& Wibberenz 1986; Litvinenko 2012a; He \& Schlickeiser 2014), and it can be written as

$$
\kappa_{\|}^{a}=\kappa_{\| 0}+T,
$$

where $T$ is the correction to $\kappa_{\| 0}$.

\section{ANALYTICAL COEFFICIENTS WITH $\Lambda(X, Y, Z, T) \neq 0$}

In general, if $\Lambda(x, y, z, t) \neq 0$, we have to obtain the coefficient formulas of the isotropic distribution function equation (see Equation (19)) with the influence of $\Lambda(\mu)$. Therefore, we need to get the terms $\partial R / \partial z$ and $\Delta_{\perp} R$ because of the Equation (20), in consequence, we have to get the formula for $R(\mu)$. By combining Equations (9) and (13) we can get the following formula

$$
\begin{aligned}
R(\mu)= & \int_{-1}^{\mu} d v \frac{e^{-M(v)}}{D_{\mu \mu}}\left[\left(\frac{\partial F}{\partial t} v+\frac{\partial}{\partial t} \int_{-1}^{v} d \rho g\right)+\frac{v}{2}\left(2 \int_{-1}^{v} d \rho \rho \frac{\partial g}{\partial z}-\int_{-1}^{1} d \mu \mu \frac{\partial g}{\partial z}\right)\right. \\
& \left.-\Delta_{\perp} F\left(\int_{-1}^{v} d \rho D_{\perp}-\frac{1}{2} \int_{-1}^{1} d \mu D_{\perp}\right)-\left(\int_{-1}^{v} d \rho D_{\perp} \Delta_{\perp} g-\frac{1}{2} \int_{-1}^{1} d \mu D_{\perp} \Delta_{\perp} g\right)\right] .
\end{aligned}
$$

With the latter equation we can get the formulas of $\partial R / \partial z$ and $\Delta_{\perp} R$ as follows

$$
\begin{aligned}
\frac{\partial R}{\partial z}= & \int_{-1}^{\mu} d v \frac{e^{-M(v)}}{D_{\mu \mu}}\left[\frac{\partial^{2} F}{\partial t \partial z} v+\frac{\partial^{2}}{\partial t \partial z} \int_{-1}^{v} g d \rho+\frac{v}{2}\left(2 \int_{-1}^{v} d \rho \rho \frac{\partial^{2} g}{\partial z^{2}}-\int_{-1}^{1} d \mu \mu \frac{\partial^{2} g}{\partial z^{2}}\right)\right. \\
& \left.-\Delta_{\perp} \frac{\partial F}{\partial z}\left(\int_{-1}^{v} d \rho D_{\perp}-\frac{1}{2} \int_{-1}^{1} d \mu D_{\perp}\right)-\left(\int_{-1}^{v} d \rho D_{\perp} \Delta_{\perp} \frac{\partial g}{\partial z}-\frac{1}{2} \int_{-1}^{1} d \mu D_{\perp} \Delta_{\perp} \frac{\partial g}{\partial z}\right)\right]
\end{aligned}
$$

and

$$
\begin{aligned}
\Delta_{\perp} R= & \int_{-1}^{\mu} d v \frac{e^{-M(v)}}{D_{\mu \mu}}\left[\Delta_{\perp} \frac{\partial F}{\partial t} v+\Delta_{\perp} \frac{\partial}{\partial t} \int_{-1}^{v} g d \rho+\frac{v}{2}\left(2 \int_{-1}^{v} d \rho \rho \Delta_{\perp} \frac{\partial g}{\partial z}-\int_{-1}^{1} d \mu \mu \Delta_{\perp} \frac{\partial g}{\partial z}\right)\right. \\
& \left.-\Delta_{\perp}^{2} F\left(\int_{-1}^{v} d \rho D_{\perp}-\frac{1}{2} \int_{-1}^{1} d \mu D_{\perp}\right)-\left(\int_{-1}^{v} d \rho D_{\perp} \Delta_{\perp}^{2} g-\frac{1}{2} \int_{-1}^{1} d \mu D_{\perp} \Delta_{\perp}^{2} g\right)\right] .
\end{aligned}
$$


From the latter equations we can find that the terms $\partial^{2} g / \partial z^{2}, \Delta_{\perp} \partial g / \partial z, \Delta_{\perp} \partial g / \partial t$ and $\Delta_{\perp}^{2} g$ need to be obtained. Then inserting the latter equations into Equation (20), we can obtain the formulas of $\eta_{1,0,0}, \eta_{0,1,0}$, $\eta_{0,2,0}$ and $\eta_{0,0,1}$.

\subsection{The analytical perpendicular diffusion coefficient with $\Lambda(\mu) \neq 0$}

From Equation (23) we can find that the perpendicular diffusion coefficient is the corresponding coefficient of the term $\Delta_{\perp} F$, and the correction to the perpendicular diffusion coefficient from $\Lambda(x, y, z, t)$ is the coefficient of the term $\Delta_{\perp} F$ in $\Lambda(x, y, z, t)$. As shown in Equation (20), the term $\Lambda(x, y, z, t)$ is the function of $\partial R / \partial z$ and $\Delta_{\perp} R$. In the formula of $\partial R / \partial z$ (see Equation (43)), the term $\Delta_{\perp} F$ does not exist. In addition, from the formula of $\Delta_{\perp} R$ (see Equation (44)) we can find that the term including $\Delta_{\perp} F$ does not exist, too. Therefore, $\Lambda(x, y, z, t)$ does not have the term $\Delta_{\perp} F$, and the correction to the perpendicular diffusion coefficient from $\Lambda(x, y, z, t)$ is zero, i.e., $\eta_{0,0,1}=0$. From Equation (28) we can find that the perpendicular diffusion coefficient can be written as

$$
\kappa_{\perp}=\kappa_{\perp}^{a}=\frac{\int_{-1}^{1} d \mu D_{\perp} e^{M(\mu)}}{\int_{-1}^{1} d \mu e^{M(\mu)}} .
$$

In general, the Fokker-Planck perpendicular diffusion coefficient $D_{\perp}$ depends on pith-angle cosine $\mu$. By using unified nonlinear transport theory (Shalchi 2010), Qin \& Shalchi (2014) developed a model of $D_{\perp}(\mu)$ to find $D_{\perp}(\mu) \propto|\mu|$. But this model describes the case for uniform background magnetic field. Maybe the weakly nonlinear theory (Shalchi et al. 2004) can be used to derive the formula of $D_{\perp}(\mu)$ with adiabatic focusing effect, but this procedure is too complicated. Therefore, so far, no mathematically tractable theory describing the relationship of $D_{\perp}$ to pitch angle cosine $\mu$ and adiabatic focusing is obtained to explore this problem. In this paper, we only obtain the latter formula of the perpendicular diffusion coefficient with the adiabatic focusing, but do not explore it in detail.

\subsection{The analytical parallel diffusion coefficient with $\Lambda(x, y, z, t) \neq 0$}

As shown in Equation (23), coefficient $\eta_{0,2,0}$ is the correction from $\Lambda(x, y, z, t)$ to the parallel diffusion coefficient. It is obvious that there is no term $\partial^{2} F / \partial z^{2}$ in $\Delta_{\perp} R$ (see Equation (44)), but there might be the term $\partial^{2} F / \partial z^{2}$ in $\partial R / \partial z$ (see Equation (43)). Therefore, the correction from $\Lambda(x, y, z, t)$ (Equation (20)) to 
the parallel diffusion coefficient should only come from

$$
-\frac{v}{2} \int_{-1}^{1} d \mu \mu e^{M(\mu)}\left[\frac{\partial R}{\partial z}-\frac{\int_{-1}^{1} d \mu \frac{\partial R}{\partial z} e^{M(\mu)}}{\int_{-1}^{1} d \mu e^{M(\mu)}}\right] .
$$

From Equation (43) we can find that only the terms $\partial^{2} g / \partial z^{2}$, i.e.,

$$
\frac{v}{2} \int_{-1}^{\mu} d v \frac{e^{-M(v)}}{D_{\mu \mu}}\left(2 \int_{-1}^{v} d \rho \rho \frac{\partial^{2} g}{\partial z^{2}}-\int_{-1}^{1} d \mu \mu \frac{\partial^{2} g}{\partial z^{2}}\right),
$$

might have the contribution to term $\partial^{2} F / \partial z^{2}$ in $\partial R / \partial z$. By operating $\partial^{2} / \partial z^{2}$ on the formula of $g(\mu$ ) (see Equation (12)), we can obtain

$$
\frac{\partial^{2} g}{\partial z^{2}}=\left(L \frac{\partial^{3} F}{\partial z^{3}}-\frac{\partial^{2} F}{\partial z^{2}}\right)\left[1-\frac{2 e^{M(\mu)}}{\int_{-1}^{1} d \mu e^{M(\mu)}}\right]+e^{M(\mu)}\left[\frac{\partial^{2} R}{\partial z^{2}}-\frac{\int_{-1}^{1} d \mu e^{M(\mu)} \frac{\partial^{2} R}{\partial z^{2}}}{\int_{-1}^{1} d \mu e^{M(\mu)}}\right] .
$$

From Equation (42), $\partial^{2} R / \partial z^{2}$ can be written as

$$
\begin{aligned}
\frac{\partial^{2} R}{\partial z^{2}}= & \int_{-1}^{\mu} d v \frac{e^{-M(v)}}{D_{\mu \mu}}\left[\frac{\partial^{3} F}{\partial t \partial z^{2}} v+\frac{\partial^{3}}{\partial t \partial z^{2}} \int_{-1}^{v} g d \rho+\frac{v}{2}\left(2 \int_{-1}^{v} d \rho \rho \frac{\partial^{3} g}{\partial z^{3}}-\int_{-1}^{1} d \mu \mu \frac{\partial^{3} g}{\partial z^{3}}\right)\right. \\
& \left.-\Delta_{\perp} \frac{\partial^{2} F}{\partial z^{2}}\left(\int_{-1}^{v} d \rho D_{\perp}-\frac{1}{2} \int_{-1}^{1} d \mu D_{\perp}\right)-\left(\int_{-1}^{v} d \rho D_{\perp} \Delta_{\perp} \frac{\partial^{2} g}{\partial z^{2}}-\frac{1}{2} \int_{-1}^{1} d \mu D_{\perp} \Delta_{\perp} \frac{\partial^{2} g}{\partial z^{2}}\right)\right]
\end{aligned}
$$

Obviously, the term $\partial^{2} F / \partial z^{2}$ does not exist on the right hand side of the latter equation. Therefore, we can find that the term $\partial^{2} F / \partial z^{2}$ on the right hand side of Equation (48) is

$$
-\frac{\partial^{2} F}{\partial z^{2}}\left[1-\frac{2 e^{M(\mu)}}{\int_{-1}^{1} d \mu e^{M(\mu)}}\right]
$$

By replacing $\partial^{2} g / \partial z^{2}$ in expression (47) with expression (50) we find the term $\partial^{2} F / \partial z^{2}$ in $\partial R / \partial z$ as

$$
-v \frac{\partial^{2} F}{\partial z^{2}} \int_{-1}^{\mu} d v \frac{e^{-M(v)}}{D_{\mu \mu}}\left\{\frac{\int_{-1}^{1} d \mu \mu e^{M(\mu)}}{\int_{-1}^{1} d \mu e^{M(\mu)}}+\int_{-1}^{v} d \rho \rho\left[1-\frac{2 e^{M(\mu)}}{\int_{-1}^{1} d \mu e^{M(\mu)}}\right]\right\} .
$$

By substituting $\partial^{2} g / \partial z^{2}$ in expression (46) with the latter expression, we can obtain the term $\partial^{2} F / \partial z^{2}$ in $\Lambda(x, y, z, t)$ as

$$
\begin{gathered}
-\frac{v^{2}}{2}\left\{\frac{\int_{-1}^{1} d \mu \mu e^{M(\mu)}}{\int_{-1}^{1} d \mu e^{M(\mu)}} \int_{-1}^{1} d \mu e^{M(\mu)} \int_{-1}^{\mu} d v \frac{e^{-M(v)}}{D_{\mu \mu}}\left[\int_{-1}^{\nu} d \rho \rho\left(1-\frac{2 e^{M(\rho)}}{\int_{-1}^{1} d \mu e^{M(\mu)}}\right)+\int_{-1}^{1} d \mu \mu \frac{e^{M(\mu)}}{\int_{-1}^{1} d \mu e^{M(\mu)}}\right]\right. \\
\left.-\int_{-1}^{1} d \mu \mu e^{M(\mu)} \int_{-1}^{\mu} d v \frac{e^{-M(v)}}{D_{\mu \mu}}\left[\int_{-1}^{v} d \rho \rho\left(1-\frac{2 e^{M(\rho)}}{\int_{-1}^{1} d \mu e^{M(\mu)}}\right)+\int_{-1}^{1} d \mu \mu \frac{e^{M(\mu)}}{\int_{-1}^{1} d \mu e^{M(\mu)}}\right]\right\} \frac{\partial^{2} F}{\partial z^{2}}
\end{gathered}
$$


so the correction coefficient $\eta_{0,2,0}$ from $\Lambda(\mu)$ to the parallel diffusion coefficient can be written as

$$
\begin{aligned}
& \eta_{0,2,0}=\frac{v^{2}}{2}\left\{\int_{-1}^{1} d \mu \mu e^{M(\mu)} \int_{-1}^{\mu} d \nu \frac{e^{-M(v)}}{D_{\mu \mu}}\left[\int_{-1}^{\nu} d \rho \rho\left(1-\frac{2 e^{M(\rho)}}{\int_{-1}^{1} d \mu e^{M(\mu)}}\right)+\int_{-1}^{1} d \mu \mu \frac{e^{M(\mu)}}{\int_{-1}^{1} d \mu e^{M(\mu)}}\right]\right. \\
& \left.-\frac{\int_{-1}^{1} d \mu \mu e^{M(\mu)}}{\int_{-1}^{1} d \mu e^{M(\mu)}} \int_{-1}^{1} d \mu e^{M(\mu)} \int_{-1}^{\mu} d \nu \frac{e^{-M(v)}}{D_{\mu \mu}}\left[\int_{-1}^{\nu} d \rho \rho\left(1-\frac{2 e^{M(\rho)}}{\int_{-1}^{1} d \mu e^{M(\mu)}}\right)+\int_{-1}^{1} d \mu \mu \frac{e^{M(\mu)}}{\int_{-1}^{1} d \mu e^{M(\mu)}}\right]\right\} .
\end{aligned}
$$

Since $\kappa_{\|}^{a}=\kappa_{\| 0}+T$ (see Equation (41)) and considering Equation (29), we can obtain

$$
\kappa_{\|}=\kappa_{\|}^{a}+\eta_{0,2,0}=\kappa_{\| 0}+T+\eta_{0,2,0}
$$

Thus, by considering $\Lambda(\mu)$ we find a new correction $T^{\prime}$ to $\kappa_{\| 0}$ as

$$
\kappa_{\|}=\kappa_{\| 0}+T^{\prime}
$$

with

$$
T^{\prime}=T+\eta_{0,2,0}
$$

Here, $T$ is the correction obtained by previous authors (see, Beeck \& Wibberenz 1986; Litvinenko 2012a; Shalchi \& Danos 2013; He \& Schlickeiser 2014), and the formula of $\eta_{0,2,0}$ is Equation (53). From Equations (22) and (23) we can find that $\eta_{0,2,0}$ is created by the term $\Lambda(\vec{x}, t)$ on the right hand side of Equation (22). The correction coming from higher-order derivative term might not be necessarily high-order small quantity relative to the correction from other terms. Therefore, we cannot arbitrarily neglect any term in the governing equation (22). In Subsection 4.5, $\eta_{0,2,0}$ will be evaluated and compared with the correction from other terms in Equation (22).

\subsection{The analytical parallel streaming coefficient with $\Lambda(x, y, z, t) \neq 0$}

From Equation (44) we can find that the term $\partial F / \partial z$ does not exist in $\Delta_{\perp} R$, however, it could exist in $\partial R / \partial z$. Therefore, the correction from $\Lambda(x, y, z, t)$ to the parallel streaming term $\partial F / \partial z$ could only come from the term

$$
-\frac{v}{2} \int_{-1}^{1} d \mu \mu e^{M(\mu)}\left[\frac{\partial R}{\partial z}-\frac{\int_{-1}^{1} d \mu \frac{\partial R}{\partial z} e^{M(\mu)}}{\int_{-1}^{1} d \mu e^{M(\mu)}}\right] .
$$

According to the formula of $\partial R / \partial z$ (see Equation (43)), the term $\partial F / \partial z$ does not exist in the expression of $\partial R / \partial z$. So that there is no term $\partial F / \partial z$ in expression (57). Therefore, there is no correction from $\Lambda(x, y, z, t)$ 
to the parallel streaming, i.e., the coefficient $\eta_{0,1,0}$ is equal to 0. In consequence, from Equation (27) the coefficient of the parallel streaming is

$$
\kappa_{1}=\kappa_{1}^{a}=v \frac{\int_{-1}^{1} d \mu \mu e^{M(\mu)}}{\int_{-1}^{1} d \mu e^{M(\mu)}} .
$$

4.4. The analytical coefficient of the first order time derivative term with $\Lambda(x, y, z, t) \neq 0$

From Equation (42) we find that $\Lambda(x, y, z, t)$ is the function of $\partial R / \partial z$ and $\Delta_{\perp} R$. Since the term $\partial F / \partial t$ does not exist in terms $\partial R / \partial z$ and $\Delta_{\perp} R$, from Equation (23) we can find that the correction from $\Lambda(x, y, z, t)$ to the first order time derivative term is 0 , i.e., $\eta_{1,0,0}=0$. Therefore, from Equation (26) we can obtain

$$
\kappa_{t}=\kappa_{t}^{a}=1
$$

\subsection{Evaluating the correction $T^{\prime}$ for model $D_{\mu \mu}=D\left(1-\mu^{2}\right)$}

In the Subsection 4.2, the parallel diffusion coefficient for the case $\Lambda(x, y, z, t) \neq 0$ has been obtained as $\kappa_{\|}=\kappa_{\| 0}+T^{\prime}$ with the correction $T^{\prime}=T+\eta_{0,2,0}$. In Appendix A we find that the limits of $T$ and $\eta_{0,2,0}$ all tend to 0 for the limit $L \rightarrow \infty$. That is, the correction $T^{\prime}$ tends to 0 for the limit $L \rightarrow \infty$. Therefore, $\kappa_{\|} \rightarrow \kappa_{\kappa} 0$ when the spatially varying background magnetic field tends to the uniform one. In the following we evaluate the correction $T^{\prime}$ for the isotropic model $D_{\mu \mu}=D\left(1-\mu^{2}\right)$.

As shown in He \& Schlickeiser (2014), for the isotropic pitch-angle scattering model $D_{\mu \mu}=D\left(1-\mu^{2}\right)$, Equation (10) can be simplified as

$$
M(\mu)=\xi(\mu+1)
$$

with

$$
\xi=\frac{v}{2 D L} .
$$

In Appendix B, by using the latter simple model the correction coefficient $\eta_{0,2,0}$ from $\Lambda(\mu)$ is evaluated. We can find that the magnitude of the lowest order of the correction $\eta_{0,2,0}$ is larger than that of the correction $T$. If $T$ needs to be considered, the correction coefficient $\eta_{0,2,0}$ from $\Lambda(\mu)$ cannot be ignored.

Inserting the quantities $T$ (Equation (B2)), $S$ (Equation (B3)) and $\eta_{0,2,0}$ (Equation (B17)) into the formula of $\kappa_{\|}$(Equation (55)), we can obtain

$$
\kappa_{\|} \approx \kappa_{\| 0}\left(1+\frac{2}{15} \xi^{2}\right)
$$


Because $\kappa_{\|}=\kappa_{\|}^{0}+T^{\prime}$, we can find

$$
T^{\prime} \approx \frac{2}{15} \xi^{2} \kappa_{\| 0}
$$

The lowest order correction of $T$ obtained by the previous authors is equal to $-\xi^{2} \kappa_{\| 0} / 15$ (see, Beeck \& Wibberenz 1986; Litvinenko 2012b; Shalchi \& Danos 2013; He \& Schlickeiser 2014). However, in this paper the lowest order correction of $T^{\prime}$ is equal to $2 \xi^{2} \kappa_{\|}^{0} / 15$. Therefore, at least for the isotropic model $D_{\mu \mu}=D\left(1-\mu^{2}\right)$ with $\xi \ll 1$, to consider the adiabatic focusing effect, the new correction $T^{\prime}$ should be used.

\section{SUMMARY AND CONCLUSION}

In this paper, by using the improved perturbation method of He \& Schlickeiser (2014) and the iteration process, we explore the influence of along-field adiabatic focusing on energetic charged particle transport. Starting from the modified linear Fokker-Planck equation with the pitch-angle scattering and perpendicular transport and adiabatic focusing effect, we obtain the governing equation of the isotropic distribution function $F(\vec{x}, t)$ with infinite terms, from which we get the coefficients of the spatial parallel and perpendicular diffusion, and the coefficient of parallel streaming term. The parallel diffusion coefficient can be written as $\kappa_{\|}=\kappa_{\| 0}+T^{\prime}$, where $\kappa_{\| 0}$ is the parallel diffusion coefficient for the uniform background magnetic field, and $T^{\prime}$ is the correction to the parallel diffusion coefficient. We also get $T^{\prime}=T+\eta_{0,2,0}$ with $T$ being the correction derived in the previous papers by ignoring the higher-order derivative terms in the isotropic distribution function equation (Beeck \& Wibberenz 1986; Litvinenko 2012a; He \& Schlickeiser 2014), and $\eta_{0,2,0}$ coming from the higher-order derivative terms obtained in this paper but ignored by the previous authors.

Moreover, for the isotropic pitch-angle scattering model $D_{\mu \mu}=D\left(1-\mu^{2}\right)(D$ is a constant) with $\xi \ll 1$, We find that the magnitude of correction coefficient $\eta_{0,2,0}$ is larger than that of the correction $T$ obtained in the previous paper. And the correction $T^{\prime}=T+\eta_{0,2,0}$ even has different sign as $T$. In the previous papers, the higher-order derivative terms shown by $\Lambda(\vec{x}, t)$ in the isotropic distribution function equation (Equation (22)) was neglected. However, in this paper we find that the higher-order derivative terms $\Lambda(\vec{x}, t)$ also can make correction to the parallel diffusion coefficients, i.e., the correction formula $\eta_{0,2,0}$. Therefore, the correction $T$ obtained in the previous papers is approximate. In addition, we find that the magnitude of $\eta_{0,2,0}$, which is to the parallel diffusion coefficient, is larger than that of $T$ derived by the previous authors. Therefore, the 
higher-order derivative term in the governing equation of isotropic distribution function cannot be arbitrarily ignored.

In addition, we obtain the formula of the perpendicular diffusion coefficient $\kappa_{\perp}$. Since there is no appropriate theory describing the relationship of the Fokker-Planck perpendicular diffusion coefficient $D_{\perp}$ to pitch-angle cosine $\mu$ and adiabatic focusing effect, we do not explore it in detail. Furthermore, we find that, from the terms ignored by the previous authors in the governing equation of $F$, the corrections to the coefficients of the spatial perpendicular diffusion, the parallel streaming, and the first order time derivative term are equal to 0 .

It is noted that $D_{\mu \mu}$ used in computing $\kappa_{\|}$in this paper as well as in previous ones does not include the adiabatic focusing effect. However, $D_{\mu \mu}$ is corrected by the adiabatic focusing effect, which is represented by the adiabatic focusing characteristic length $L$, i.e., $D_{\mu \mu}=D_{\mu \mu}(\mu, L)$ (Tautz et al 2014). In fact, pitch-angle diffusion coefficient $D_{\mu \mu}$ and perpendicular diffusion coefficient $D_{\perp}$ are related to each other (e.g., Shalchi 2009a). Therefore, $D_{\perp}$ should also be corrected by the adiabatic focusing effect, i.e., $D_{\perp}=D_{\perp}(\mu, L)$. So far, no mathematically tractable theory describing the relationship of $D_{\mu \mu}$ and $D_{\perp}$ to pitch angle cosine $\mu$ and adiabatic focusing can be used to explore this problem. In addition, the correction formula $\eta_{0,2,0}$ (Equation (53)) is too complicated and it is very difficult to compute the correction. Our purpose is to show that in order to explore the correction effect the higher-order derivative terms in the governing equation cannot be neglected.

Compared with the method used in this paper, Legendre polynomial expansions to solve the z-integrated Fokker-Planck equation is a more systematic approach. But the recursive relation of different order coefficients of the expansion series cannot be obtained. In addition, we have not found a method to transform the z-integrated Fokker-Planck equation into another form, from which one to one relationship of coefficients of the expansion series can be obtained. In the future we will continue to investigate this problem.

By using different truncating methods and transformations, from the modified Fokker-Planck equation one can obtain the diffusion equation, the telegraph equation, or other equations. Telegraph equation is also very important. As another project, we are exploring the telegraph equation derived from the modified Fokker-Planck equation by employing the method of He \& Schlickeiser (2014). 
In the future, to obtain more accurate analytical formulas of the spatial parallel and perpendicular diffusion coefficients, we plan to get the mathematical tractable formulas of $D_{\mu \mu}(L, \mu)$ and $D_{\perp}(L, \mu)$. In addition, we plan to numerically compute the correction $T^{\prime}$ and compare it with $T$ for different turbulence models and conditions. In addition, The analysis to the different length scales and time scales in the problem is also important. We will explore this problem by using scale analysis and dimensional analysis in the future.

We are partly supported by grants NNSFC 41574172 and NNSFC 41874206.

\section{APPENDIX}

\section{A. PARALLEL DIFFUSION COEFFICIENT $\kappa_{\|}$FOR THE LIMIT $L \rightarrow \infty$}

From Equations (55) and (56), the parallel diffusion coefficient $\kappa_{\|}$with adiabatic focusing effect can be written as $\kappa_{\|}=\kappa_{\| 0}+T^{\prime}=\kappa_{\| 0}+T+\eta_{0,2,0}$ with the parallel diffusion coefficient for uniform background field $\kappa_{\| 0}$, the correction $T$ obtained in previous papers, and the correction formula $\eta_{0,2,0}$ derived in this paper. If adiabatic focusing characteristic length $L$ tends to infinity, i.e., $\xi$ tends to zero, the parallel diffusion coefficient $\kappa_{\|}$should tend to the uniform field parallel diffusion coefficient $\kappa_{\| 0}$, i.e., $\lim _{L \rightarrow \infty} T^{\prime}=0$. Therefore, $\lim _{L \rightarrow \infty} \eta_{0,2,0}=0$ and $\lim _{L \rightarrow \infty} T=0$. In the following, we prove the above limits.

The correction coefficient $\eta_{0,2,0}$ (see Equation (53)) is the function of quantity $M(\mu)$ (see Equation (10)). So that we have to explore $M(\mu)$ for the limit $L \rightarrow \infty$, i.e., $\lim _{L \rightarrow \infty} M(\mu)$. If the integral $\int_{-1}^{\mu} d v\left(1-v^{2}\right) / D_{\mu \mu}(v)$ in $M(\mu)$ (see Equation (10)) is convergent, we can find $\lim _{L \rightarrow \infty} M(\mu)=0$. Accordingly, the limit

$$
\begin{gathered}
\lim _{L \rightarrow \infty} e^{M(\mu)}=1, \\
\lim _{L \rightarrow \infty} e^{-M(\mu)}=1 .
\end{gathered}
$$

can be obtained. Therefore, we can find the following relation

$$
\lim _{L \rightarrow \infty} \frac{\int_{-1}^{1} d \mu \mu e^{M(\mu)}}{\int_{-1}^{1} d \mu e^{M(\mu)}}=0
$$

Similarly, the following formula can be found

$$
\lim _{L \rightarrow \infty} \int_{-1}^{\mu} d v v\left(1-\frac{2 e^{M(v)}}{\int_{-1}^{1} d \mu e^{M(\mu)}}\right)=0 .
$$


By inserting Equations (A3) and (A4) into Equation (53), we can easily find that $\lim _{L \rightarrow \infty} \eta_{0,2,0}=0$.

Secondly, we investigate $\lim _{L \rightarrow \infty} \kappa_{\|}^{a}$. We already find that $\lim _{L \rightarrow \infty} M(\mu)=0$ in the above paragraph. Using $\lim _{L \rightarrow \infty} e^{M(\mu)}=1+\lim _{L \rightarrow \infty} M(\mu)$, from Equation (39) the following equation can be obtained

$$
\lim _{L \rightarrow \infty} \kappa_{\|}^{a}=v L \frac{\lim _{L \rightarrow \infty} \int_{-1}^{1} d \mu \mu M(\mu)}{2+\lim _{L \rightarrow \infty} \int_{-1}^{1} d \mu M(\mu)} .
$$

By using integration in parts and the definition of $M(\mu)$ (see Equation (10)), we can obtain

$$
\int_{-1}^{1} d \mu \mu M(\mu)=\frac{v}{4 L} \int_{-1}^{1} d \mu \frac{\left(1-\mu^{2}\right)^{2}}{D_{\mu \mu}(\mu)}
$$

Inserting Equations (10) and (A6) into Equation (A5) yields

$$
\lim _{L \rightarrow \infty} \kappa_{\|}^{a}=\frac{v^{2}}{4} \frac{\lim _{L \rightarrow \infty} \int_{-1}^{1} d \mu\left(1-\mu^{2}\right)^{2} / D_{\mu \mu}(\mu)}{2+\lim _{L \rightarrow \infty} U / L}
$$

with

$$
U=\frac{v}{2} \int_{-1}^{1} d \mu \int_{-1}^{\mu} d v\left(1-v^{2}\right) / D_{\mu \mu}(v)
$$

If $U$ is finite, the following can be obtained

$$
\lim _{L \rightarrow \infty} \frac{U}{L}=0
$$

and Equation (A7) becomes

$$
\lim _{L \rightarrow \infty} \kappa_{\|}^{a}=\frac{v^{2}}{8} \int_{-1}^{1} d \mu \frac{\left(1-\mu^{2}\right)^{2}}{D_{\mu \mu}} .
$$

The latter formula is identical with the parallel diffusion coefficient $\kappa_{\| 0}$ for uniform background magnetic field (Jokipii 1966; Hasselmann \& Wibberenz 1968; Earl 1974; Shalchi 2006). So, $\lim _{L \rightarrow \infty} T=0$. Therefore, we find $\lim _{L \rightarrow \infty} \kappa_{\|}=\kappa_{\| 0}$, i.e., $\lim _{L \rightarrow \infty} T^{\prime}=0$.

\section{B. THE CORRECTION COEFFICIENT $\eta_{0,2,0}$ FROM $\Lambda(\mu)$ FOR THE ISOTROPIC MODEL$$
D_{\mu \mu}=D\left(1-\mu^{2}\right)
$$

Here, by employing the isotropic model $D_{\mu \mu}=D\left(1-\mu^{2}\right)$ we approximately evaluate $\eta_{0,2,0}$. After inserting Equation (60) into Equation (53) we can get

$$
\begin{aligned}
\eta_{0,2,0}= & \frac{v^{2}}{2}\left\{\int_{-1}^{1} d \mu \mu e^{\xi \mu} \int_{-1}^{\mu} d v \frac{e^{-\xi \nu}}{D_{\mu \mu}}\left[\frac{v^{2}-1}{2}-2 \frac{\int_{-1}^{v} d \rho \rho e^{\xi \rho}}{\int_{-1}^{1} d \mu e^{\xi \mu}}+\frac{\int_{-1}^{1} d \mu \mu e^{\xi \mu}}{\int_{-1}^{1} d \mu e^{\xi \mu}}\right]\right. \\
& \left.-\frac{\int_{-1}^{1} d \mu \mu e^{\xi \mu}}{\int_{-1}^{1} d \mu e^{\xi \mu}} \int_{-1}^{1} d \mu e^{\xi \mu} \int_{-1}^{\mu} d v \frac{e^{-\xi \nu}}{D_{\mu \mu}}\left[\frac{v^{2}-1}{2}-2 \frac{\int_{-1}^{1} d \rho \rho e^{\xi \rho}}{\int_{-1}^{1} d \mu e^{\xi \mu}}+\frac{\int_{-1}^{1} d \mu \mu e^{\xi \mu}}{\int_{-1}^{1} d \mu e^{\xi \mu}}\right]\right\} .
\end{aligned}
$$


The correction $T$ to the parallel diffusion coefficient $\kappa_{\| 0}$ has been obtained in the previous papers (see, Beeck \& Wibberenz 1986; Litvinenko 2012b; Shalchi \& Danos 2013; He \& Schlickeiser 2014) as

$$
T=\kappa_{\| 0} S
$$

with

$$
S=-\frac{1}{15} \xi^{2}+\frac{2}{315} \xi^{4}+\cdots
$$

To proceed, by employing the latter formula we can get

$$
\frac{\int_{-1}^{1} d \mu \mu e^{\xi \mu}}{\int_{-1}^{1} d \mu e^{\xi \mu}}=\frac{1+S}{3} \xi
$$

By inputting Equation (B4) into Equation (B1), we can obtain

$$
\eta_{0,2,0}=\frac{v^{2}}{2}\left[Y_{2}-\frac{1+S}{3} \xi Y_{1}\right]
$$

with

$$
\begin{aligned}
Y_{1} & =\int_{-1}^{1} d \mu e^{\xi \mu} \int_{-1}^{\mu} d v Z(v), \\
Y_{2} & =\int_{-1}^{1} d \mu \mu e^{\xi \mu} \int_{-1}^{\mu} d v Z(v), \\
Z(\mu) & =\frac{e^{-\xi \nu}}{D_{\mu \mu}}\left[\frac{v^{2}-1}{2}-2 \frac{\int_{-1}^{v} d \rho \rho e^{\xi \rho}}{\int_{-1}^{1} d \mu e^{\xi \mu}}+\frac{1+S}{3} \xi\right] .
\end{aligned}
$$

By integration in parts for Equations (B6) and (B7), we can obtain

$$
\begin{aligned}
& Y_{1}=\frac{e^{\xi}}{\xi} \int_{-1}^{1} d \mu Z(\mu)-\frac{1}{\xi} \int_{-1}^{1} d \mu e^{\xi \mu} Z(\mu), \\
& Y_{2}=\frac{e^{\xi}}{\xi} \int_{-1}^{1} d \mu Z(\mu)-\frac{1}{\xi} \int_{-1}^{1} d \mu e^{\xi \mu} Z(\mu) \mu-\frac{1}{\xi} Y_{1} .
\end{aligned}
$$

After inserting Equations (B9) and (B10) into Equation (B5), we can get

$$
\eta_{0,2,0}=\frac{v^{2}}{2}\left[e^{\xi}\left(\frac{1}{\xi}-\frac{1}{\xi^{2}}-\frac{1+S}{3}\right) \int_{-1}^{1} d \mu Z(\mu)-\frac{1}{\xi} \int_{-1}^{1} d \mu \mu e^{\mu \xi} Z(\mu)+\left(\frac{1}{\xi^{2}}+\frac{1+S}{3}\right) \int_{-1}^{1} d \mu e^{\mu \xi} Z(\mu)\right] .
$$

For $\xi \ll 1$, by using the following equations

$$
\begin{aligned}
& e^{\xi}=1+\xi+\frac{1}{2} \xi^{2}+\frac{1}{6} \xi^{3}+\frac{1}{24} \xi^{4}+\cdots \\
& e^{\mu \xi}=1+\mu \xi+\frac{1}{2}(\mu \xi)^{2}+\frac{1}{6}(\mu \xi)^{3}+\frac{1}{24}(\mu \xi)^{4}+\cdots
\end{aligned}
$$


we find that Equation (B11) becomes

$$
\eta_{0,2,0} \approx \frac{v^{2}}{2}\left[\frac{1}{2} \int_{-1}^{1} d \mu Z(\mu)\left(1-\mu^{2}\right)+\frac{\xi}{3} \int_{-1}^{1} d \mu Z(\mu) \mu\left(1-\mu^{2}\right)+\frac{\xi^{2}}{24} \int_{-1}^{1} d \mu Z(\mu)\left(1-\mu^{2}\right)\left(3 \mu^{2}-1\right)\right] .
$$

Similarly, employing Equations (B12) and (B13) with $\xi \ll 1$, formula (B8) can be simplified as

$$
Z(\mu) \approx \frac{1}{D_{\mu \mu}(\mu)}\left[-\frac{\mu^{3}}{3} \xi+\left(\frac{\mu^{2}-1}{12}-\frac{\mu^{4}-1}{8}+\frac{\mu^{4}}{3}\right) \xi^{2}\right] .
$$

By combining Equations (B14) and (B15), we can find

$$
\eta_{0,2,0} \approx \frac{v^{2}}{30 D} \xi^{2}
$$

In addition, because of formula $\kappa_{\| 0}=v^{2} /(6 D)$ (see, e.g., Shalchi 2009a), the latter equation becomes

$$
\eta_{0,2,0} \approx \frac{1}{5} \xi^{2} \kappa_{\| 0}
$$

\section{REFERENCES}

Beeck, J., \& Wibberenz, G. 1986, ApJ, 311, 437

Bieber, J. W., \& Burger, R. A. 1990, ApJ, 348, 597

Dwyer, J. R., Mason, G. M., Mazur, J. E., Jokipii, J. R., von Rosenvinge, T. T., \& Lepping, R. P. 1997, ApJ, 490, L115

Earl, J.A. 1974, ApJ, 193, 231

Earl, J. A. 1976, ApJ, 205, 900

Hasselmann, K., \& Wibberenz, G., Z. Geophys., 34, 353

Hauff, T., \& Jenko, F. 2008, PhPl, 15, 112307

He, H.-Q., \& Schlickeiser, R. 2014, ApJ, 792, 85

Jokipii, J. R. 1966, ApJ, 146, 480

Kóta, J. 2000, J. Geophys. Res., 105, 2403

Kunstmann, J. E. 1979, ApJ, 229, 812

Litvinenko, Y. E. 2012a, ApJ, 752, 16
Litvinenko, Y. E. 2012b, ApJ, 745, 62

Litvinenko, Y. E., \& Schlickeiser, R. 2013, A\&A, 554, A59

Matthaeus, W. H., Goldstein, M. L., \& Roberts, D. A. 1990, J. Geophys. Res., 95, 20673

Matthaeous, W.H., Gray, P.C., Pontius, D.H. Jr, \& Bieber, J.W. 1995, PhRvL, 75, 2136

Matthaeus, W. H., Qin, G., Bieber, J. W., \& Zank, G. P. 2003, ApJ, 590, L53

Qin, G., Matthaeus, W. H. \& Bieber, J. W. 2002 ApJ, 578, L117

Qin, G. 2007, ApJ, 656, 217

Qin, G., \& Shalchi, A. 2014, Applied Physics Research, 6, 1

Qin, G., \& Zhang, L.-H. 2014, ApJ, 787, 12 
Roelof, E. C. 1969, in Lectures in High Energy

Astrophysics, ed. H. Ögelmann \& J. R.

Wayland(NASA SP-199: Washington, DC: NASA), 111

Schlickeiser, R. 2002, Cosmic Ray Astrophysics

(Berlin: Springer)

Schlickeiser, R., Dohle, U., Tautz, R.C., \& Shalchi, A. 2007, ApJ, 661, 185

Schlickeiser, R., \& Jenko, F. 2010, J. Plasma Phys., 76, 317

Schlickeiser, R., \& Shalchi, A. 2008, ApJ, 686, 292

Shalchi, A., Bieber, J. W., Matthaeus, W. H., \& Qin, G. 2004, ApJ, 616, 617

Shalchi, A. 2006, A\&A, 448, 809

Shalchi, A. 2009a, Nonlinear Cosmic Ray Diffusion Theories, Astrophysics and Space Science Library, Vol. 362 (Berlin: Springer)

Shalchi, A. 2009b, J. Phys. G: Nucl. Part. Phys., 36, 025202
Shalchi, A. 2010, ApJL, 720, L127

Shalchi, A. 2011, ApJ, 728, 113

Shalchi, A., Bieber, J. W., Matthaeus, W. H., \& Schlickeiser, R. 2006, ApJ, 642, 230

Shalchi, A., \& Danos, R. J. 2013, ApJ, 765, 153

Shalchi, A., \& Kourakis, I. 2007, Phys. Plasmas, 14, 092903

Shalchi, A., \& Qin, G. 2010, Ap\&SS, 330, 279

Shalchi, A., \& Schlickeiser, R. 2005, ApJ, 626, L97

Tautz, R. C., Shalchi, A., \& Dosch, A. 2014, ApJ, 794, 138

Wang, J.-F., Qin, G., Ma, Q.-M., Song, T., \& Yuan, S.-B. 2017a, Phys. Plasmas, 24, 082901

Wang, J.-F., Qin, G., Ma, Q.-M., Song, T., \& Yuan, S.-B. 2017b, ApJ, 845, 112

Wang, Y., \& Qin, G. 2016, ApJ, 820, 61

Zhang, M., Jokipii, J. R., McKibben, R. B., 2003, ApJ, 595,493 